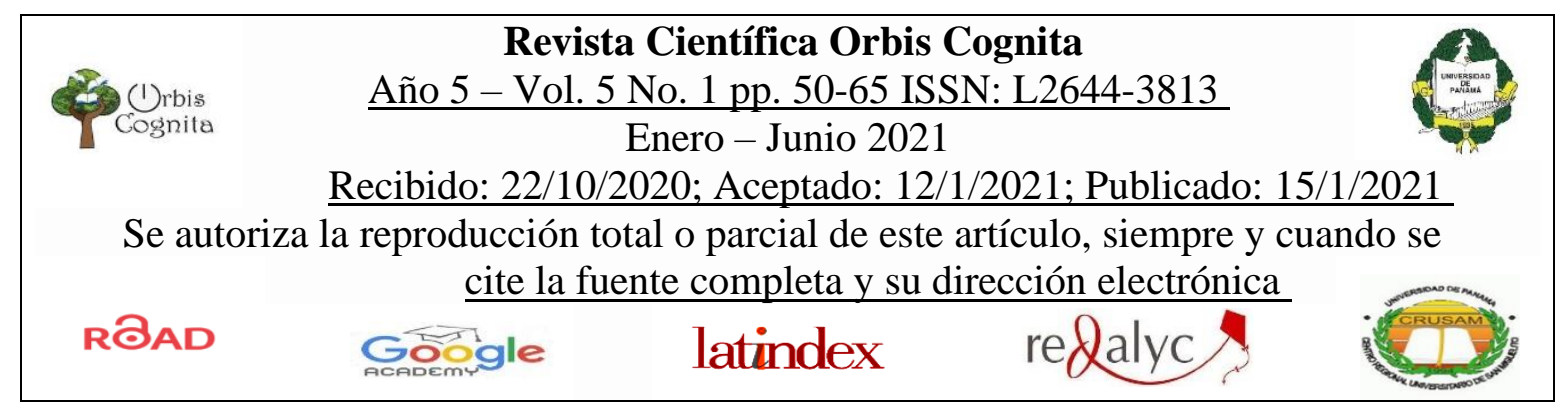

Estilos de aprendizaje en los estudiantes de inglés de acuerdo al género.

Learning styles of English students according to the gender.

\author{
Víctor Manuel López-Baloy \\ Universidad de Panamá, Facultad de Humanidades, Panamá. \\ lopezbaloy@gmail.com https://orcid.org/0000-0002-6295-0138
}

\title{
RESUMEN
}

Este artículo constituye el resultado de una investigación sobre los estilos de aprendizaje del idioma inglés desde una perspectiva de género en los estudiantes de segundo año de Derecho y Ciencias Políticas de la Universidad de Panamá (Ciudad Universitaria Octavio Méndez Pereira). El objetivo de esta investigación fue constatar si hay diferencia significativa en los estilos de aprendizaje del idioma inglés según género entre estudiantes que cursan la asignatura Lenguaje y comunicación del idioma inglés. Para la recolección de la información se utilizó el cuestionario preparado por Felder y Silverman, y se consideraron los estilos de aprendizaje activo-reflexivo, sensorial-intuitivo, visual-verbal y secuencial-global. Además, se tomó en cuenta la variable basada en los estilos de aprendizaje del idioma inglés de preferencia por los estudiantes. La metodología utilizada para la obtención de los resultados fue la hoja de calificación y registro del modelo de Felder y Silverman. Los resultados indican que hay una diferencia en algunos estilos de aprendizaje del idioma inglés entre hombres y mujeres. El estilo activo-reflexivo en varones dio como resultado un $64 \%$, mientras que las estudiantes obtuvieron un 36\%; en el de aprendizaje visual-verbal, los hombres presentaron mayor preferencia con un $76 \%$ y el sexo femenino obtuvo un $24 \%$; ambos grupos obtuvieron un $50 \%$ en el estilo de aprendizaje sensitivo-intuitivo; y en lo que respecta al aprendizaje secuencia-global, el sexo masculino obtuvo un 54\% y las mujeres, un $46 \%$. El aporte de este 
trabajo está orientado a mejorar la práctica de la enseñanza-aprendizaje del idioma inglés a nivel superior.

PALABRAS CLAVE género, estilos de aprendizaje, estilo activo-reflexivo, sensorialintuitivo, visual-verbal, secuencial-global

\begin{abstract}
This article is the result of an investigation carried out on the learning styles of the English language from a gender perspective in the second year students of the Law and Political Sciences career at the University of Panama, Central Campus. The objective of the investigation was to verify if there is a significant difference in the learning styles of the English language according to gender among students that study the subject of English and communication. The questionnaire prepared by Felder and Silverman was used for the collection of information, such as active-reflective, sensory-intuitive, visual-verbal, and sequential-global learning styles. In addition, the variable based on students' preferred English learning styles was taken into account. The methodology used to obtain the results was Felder and Silverman's model scoring and recording sheet. The results indicate that there is a difference in some English learning styles between men and women. The activereflective style in males resulted in $64 \%$, females obtained $36 \%$. In the visual-verbal learning style, males had a greater preference for this learning style at $76 \%$; females scored $24 \%$; in the sensory-intuitive learning style, both groups scored 50\%. For global sequencing learning, men scored $54 \%$, while women scored $46 \%$. The contribution of this work is aimed at improving the practice of teaching and learning the English language at a higher level.
\end{abstract}

KEYWORDS gender, learning styles, active-reflexive style, sensory-intuitive, visualverbal, sequential-global

\title{
INTRODUCCIÓN
}

A pesar de la dificultad de profundizar en las necesidades y motivaciones de cada ser humano en el proceso de adquisición del conocimiento, investigar sobre el estilo de aprendizaje en 
los estudiantes de Lenguaje y comunicación en inglés fue una inquietud que llevó a conocer las estrategias y habilidades cognitivas que utilizan los alumnos de la Licenciatura en Derecho y Ciencias Políticas en la adquisición del idioma inglés. Esta inquietud obedece, más que todo, a que los contenidos del programa de la asignatura Lenguaje y comunicación en inglés están contemplados en los programas de inglés de casi todos los bachilleratos a nivel secundario que ofrece el Ministerio de Educación en Panamá y, a pesar de ello, muchos estudiantes reprueban la asignatura. Esto representa una problemática a nivel superior en la adquisición de este idioma.

El objetivo central de este proyecto fue conocer los estilos de aprendizaje que utilizan los estudiantes en la adquisición del idioma inglés y una segunda variable fue indagar si existía una diferencia en los estilos de aprendizaje de esta lengua desde la perspectiva de género. En función de los resultados de este proyecto, se proponen recomendaciones de intervención docente para la toma de decisiones en el planeamiento didáctico de la enseñanza del inglés en beneficio de los estudiantes a nivel superior, y se espera que estas recomendaciones puedan replicarse en otros cursos de inglés impartidos en diferentes facultades de la Universidad de Panamá.

\section{Antecedentes teóricos}

El proceso de aprendizaje de una lengua extranjera no se da de manera lineal ni acumulativa, en el sentido de solo proporcionar información y conocimiento de cualquier lengua en especial; por el contrario, es un proceso de construcción de conocimientos y destrezas lingüísticas que permiten que el estudiante desarrolle el aprendizaje a través de la 
internalización, asimilación, acomodación, integración, uso y aplicación de una segunda lengua en diferentes contextos de acuerdo a las necesidades reales del aprendiz.

Con el propósito de facilitar el concepto de estilos de aprendizaje, se hizo una revisión bibliográfica extensiva de muchos autores relacionados con el tema. En este sentido, Piaget (1969) sostiene que el aprendizaje de una lengua constituye un proceso de construcción de conocimientos en el que estos se interiorizan y, junto con las representaciones de los estudiantes, desempeñan un papel fundamental en la asimilación, aceptación y el procesamiento de los nuevos conocimientos lingüísticos; así, de manera progresiva y a través de los distintos estilos de aprendizaje de cada individuo, el aprendizaje de un idioma permite la adquisición y generación de habilidades lingüísticas en el idioma aprendido (Piaget en Villanueva y Navarro, 1997).

Los estilos de aprendizaje corresponden a modelos teóricos, actúan como horizontes de la interpretación en la medida en que permiten establecer el acercamiento mayor o menor de la actuación de un sujeto a un estilo de aprendizaje. Estos estilos se caracterizan por un haz de estrategias de aprendizaje que se dan de manera correlacionada en función del aspecto cognitivo de cada individuo; por consiguiente, la enseñanza de la asignatura Lenguaje y comunicación en inglés en la educación superior debe fortalecer las destrezas lingüísticas del idioma y, por tanto, el docente de la asignatura debe manejar los distintos estilos de aprendizaje que ayudan a visualizar y descubrir las diferentes formas de representaciones mentales en que aprende el discente.

No se puede dividir a la población estudiantil en un conjunto de categorías: aprendices visuales y auditivos; en otras palabras, no hay que encasillar a las personas u ofrecerles solo una forma o estilo de aprendizaje, ya que todos somos capaces de aprender en diversos 
estilos. En esta línea, Canfield y Klimek (1977) agregan que la educación tiene que ver con la gente, de tal manera que el proceso de educación está relacionado con la evocación de la esencia transpersonal desde el mundo interior, y el desarrollo de la personalidad; lo cual involucra cuerpo, emociones, mente, imaginación y voluntad (Canfield y Klimek en Salas, 2008); por consiguiente, el acto de enseñar está relacionado con la diversidad humana; ya que no todos aprenden de la misma manera.

Las estrategias de enseñanza del docente deben estar en función de la diversidad estudiantil, lo que conlleva a comprender y adecuar los diferentes estilos de aprendizaje y poder mediar la información que llega al estudiante mediante la relación sujeto y no objeto de aprendizaje, más aun cuando se trata de un idioma que no es el materno, y el estudiante tiene que aprender a usar diferentes puntos de articulaciones que no usa en su lengua materna para poder articular sonidos que le permitan comunicarse en otro idioma. Elsner (2016) sostiene que los profesores que enseñan una lengua extranjera deben tomar en cuenta los estilos de aprendizaje del estudiante para garantizar el éxito en el proceso de enseñanza del idioma inglés y, así, ofrecer una gama de estrategias de enseñanza que permita que el aprendizaje sea significativo.

Para Brown (1994), el estilo de aprendizaje se refiere a tendencias o preferencias dentro de cada individuo y pueden considerarse un rasgo cognitivo, afectivo y fisiológico que es un indicador relativamente estable de cómo los alumnos perciben, interactúan y responden al entorno de aprendizaje. El estilo de aprendizaje de las personas está determinado por la forma en que internalizan su entorno total, y dado que el proceso de internalización no es solamente cognitivo, se pueden encontrar distintos dominios (físico, afectivo y cognitivo) que se funden en los estilos de aprendizaje. Reinert (1976) y Keefe (1979) definen los estilos de aprendizaje 
desde una perspectiva neurológica: son la forma en la que el individuo es adiestrado para aprender con mayor eficacia, procesar, comprender, recordar y ser capaz de usar la nueva información (Reinert y Keefe en Wai y otros, 2015).

Wallinton (2009) sostiene que uno de los descubrimientos más tempranos e interesantes que hicieron los neurocientíficos con imágenes funcionales fue la diferencia en la forma en que los cerebros masculinos y femeninos tienden a procesar el lenguaje. Los cerebros masculinos tienden a procesar el lenguaje en la zona izquierda, mientras que la mayoría de los cerebros femeninos procesan el lenguaje en ambos hemisferios. Esto implica que la información viaje entre los dos hemisferios cerebrales de manera más eficiente en las mujeres que en los hombres. La combinación de comunicaciones de doble hemisferio puede explicar que la mayoría de las niñas adquieran el lenguaje hablado de manera más fácil y rápida que la mayoría de los niños. Sin embargo, el debate sobre la diferencia entre géneros es continuo. Crisler (2010) afirma que, aunque las áreas de asociación auditiva del hemisferio izquierdo (el pleno temporal, por ejemplo) son más grandes en los hombres, no hay diferencia en el volumen de las áreas del hemisferio izquierdo más derecho. Cramon (2002), por su parte, indica, debido a evidencia en varios informes, que el procesamiento del lenguaje está más lateralizado en hombres que en mujeres (Cramon en Chriler, 2010). Booth (2002), gracias a patrones de activación del cerebro en niños y niñas que fueron examinados durante tareas de percepción del lenguaje visual y auditivo, sostiene que los patrones de activación bilateral en las áreas de procesamiento del lenguaje de las niñas se correlacionan con su precisión de rendimiento en la tarea sin importar si son visuales o auditivas e indica, en el caso de los niños, que los patrones de activación cerebral de precisión de las tareas dependen de la modalidad de las tareas; se llegó a la conclusión de que las niñas se basan en una 'red de 
lenguaje supramodal', mientras que los niños procesan las palabras visuales y auditivas de manera diferente (Booth en Chriler, 2010).

Felder y Silverman (1988) conciben los estilos de aprendizaje como forma independiente, cognitiva, continua, cada una anclada por diferentes aspectos: una primera dimensión identifica cómo un estudiante prefiere procesar la información y el conocimiento, proceso que se da a través de la actividad física, discusión o introspección; por otro lado, el modo sensorial en el que un estudiante prefiere recibir información y conocimiento, el cual puede ser verbal, de manera escrita, a través de ecuaciones matemáticas o visualmente (imágenes, gráficos, videos y demostraciones); en un tercer aspecto, el tipo de información que un estudiante percibe de manera más sencilla, como experiencias intuitivas, corazonadas, perspectivas, posibilidades o experiencias sensoriales basadas en el exterior; finalmente, en cómo un alumno adquiere conocimientos de manera secuencial, poco a poco o de manera holística.

Felder y Silverman (1988) concluyen en que el índice de estilos de aprendizaje tiene dos implicaciones principales:

1. proporcionar orientación a los instructores sobre la diversidad de estilos de aprendizaje dentro de sus clases y ayudarles a diseñar instrucciones que aborden las necesidades de aprendizaje de todos sus estudiantes;

2. y brindar a los estudiantes información individual sobre sus posibles fortalezas y debilidades de aprendizaje, de tal manera que se identifiquen las preferencias de estilos de aprendizaje de cada estudiante. 


\section{MATERIALES Y MÉTODOS}

La investigación se realizó considerando dos enfoques: cualitativo y cuantitativo. El enfoque cualitativo de la investigación se da en función de conocer e identificar los diferentes estilos de aprendizaje en los estudiantes masculinos y femeninos que toman la asignatura Lenguaje y comunicación en inglés. Cuantitativamente, se indagó, mediante un cuestionario de 44 preguntas elaborado por Felder y Silverman para conocer los diferentes estilos de aprendizaje del individuo (estos resultados son presentados en forma de tabla y gráficos para una mejor comprensión).

Esta investigación es de tipo descriptivo-interpretativo. Pretende identificar los estilos de aprendizaje de los estudiantes masculinos y femeninos a nivel superior en relación al aprendizaje del idioma inglés y toma como referencia las teorías de destacados autores que han escrito sobre el tema.

La recolección de la información se hizo mediante las siguientes técnicas e instrumentos:

1. Se utilizó, para la interpretación de los datos obtenidos, el cuestionario de Felder y Silverman (44 preguntas).

2. Se hizo una hoja de calificación en la que se registró cada pregunta con dos posibles respuestas ( $\mathrm{a}$ y b).

3. Se hizo la sumatoria de cada respuesta en función de la opción marcada por el participante.

4. Se registró cada una de las preguntas, desde la 1 hasta la 44.

5. Se sumó cada columna anotando el resultado de esta. 
6. En la hoja de perfil de los resultados, se restó el resultado menor al mayor y se le asignó la letra correspondiente ( a o b). Esta metodología se utilizó en la tabulación de las preguntas, tanto del grupo encuestado de hombres como del de mujeres.

7.Se utilizó el programa de Excel para sacar los porcentuales de cada grupo.

8.Se elaboraron cuatro gráficos comparativos sobre los diferentes estilos de aprendizaje del idioma inglés de hombres y mujeres.

\section{RESULTADOS}

Tabla 1. Estilos de aprendizaje de los estudiantes masculinos y femeninos a nivel superior en relación con el aprendizaje del idioma inglés

\begin{tabular}{c|c|c|c|c}
\hline Grupo masculino & Activo-reflexivo & $\begin{array}{c}\text { Sensorial- } \\
\text { intuitivo }\end{array}$ & Visual-verbal & Secuencial-global \\
\hline 37 & 83 & 95 & 161 & 123 \\
\hline Grupo femenino & 46 & 96 & 50 & 106 \\
\hline
\end{tabular}

Fuente: Encuesta aplicada a los estudiantes de Derecho y Ciencias políticas de la Universidad de Panamá, Campus Central.

Gráfica 1. Datos porcentuales del estilo de aprendizaje activo-reflexivo 


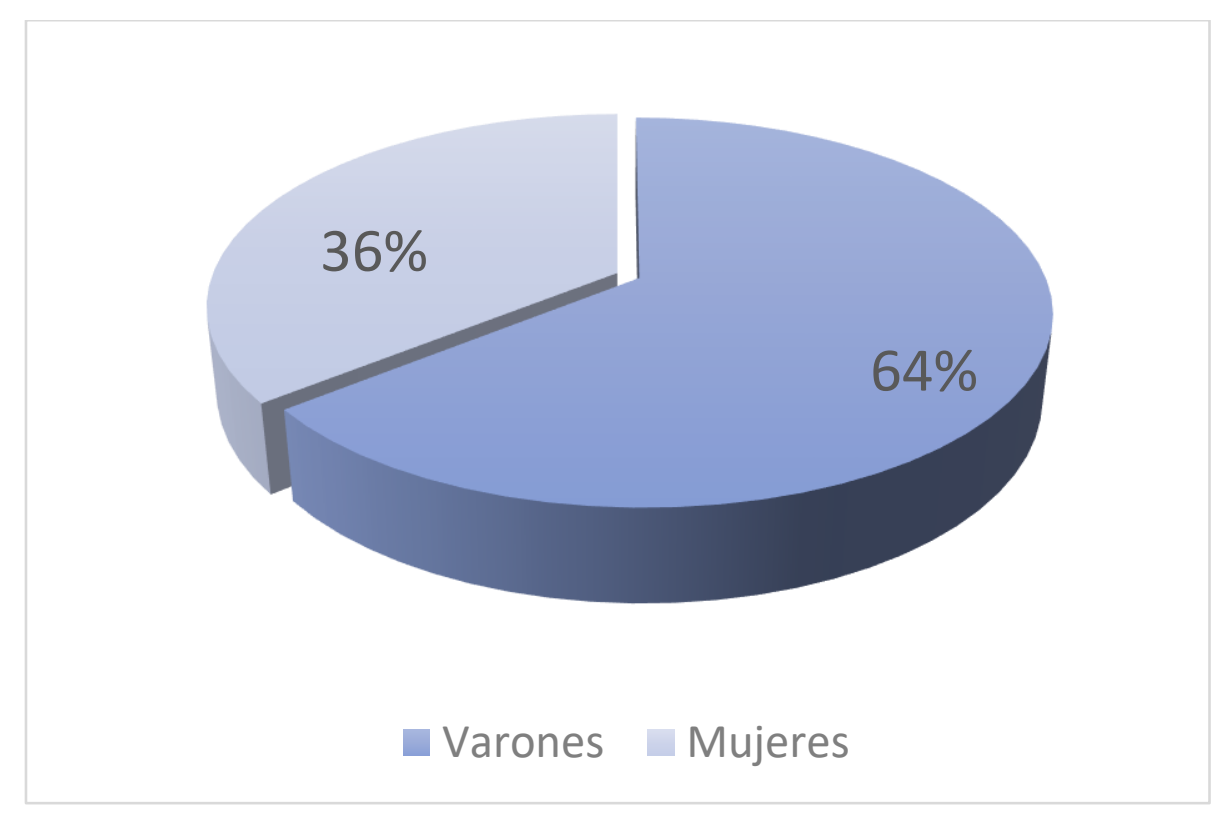

Fuente: Encuesta aplicada por el autor, basada en el cuestionario de Felder y Silverman.

Con relación a los resultados hallados en función del estilo de aprendizaje activo-reflexivo del idioma inglés, se evidencia un $64 \%$ correspondiente a los hombres en comparación con el grupo femenino, en donde se evidencia un $36 \%$.

Cabe señalar que, al observar detalladamente los porcentajes obtenidos en cuanto al estilo de aprendizaje activo-reflexivo, existe una pequeña diferencia entre este estilo de aprendizaje entre alumnos. Esto indica que ambos géneros prefieren el estilo de aprendizaje activoreflectivo, debido a que pueden procesar los contenidos del idioma inglés mediante tareas y actividades en donde ellos puedan practicar el idioma a través de la reflexión, participación y aplicación del idioma en contextos específicos.

Gráfica 2. Datos porcentuales del estilo de aprendizaje sensitivo-intuitivo 


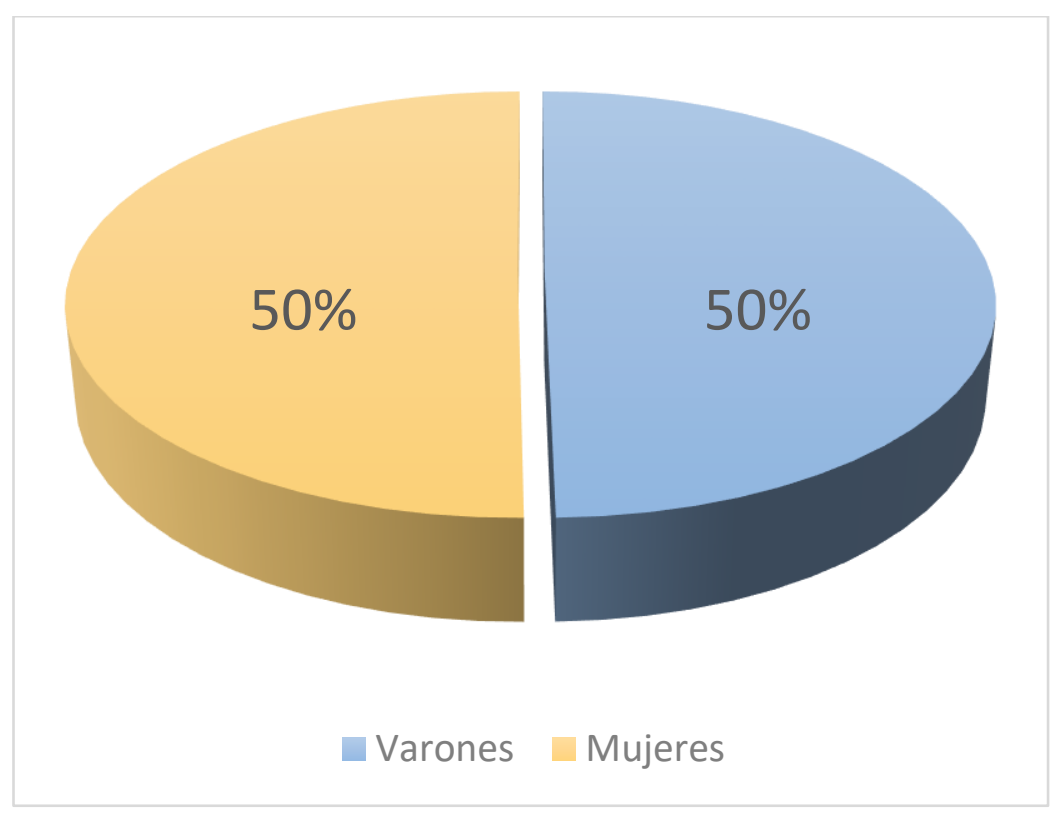

Fuente: Encuesta aplicada por el autor, basada en el cuestionario de Felder y Silverman.

De acuerdo con la gráfica 2, se observa que el $50 \%$ de las estudiantes de la asignatura Lenguaje y comunicación en inglés se inclinan por el estilo de aprendizaje sensitivo-intuitivo. Asimismo, la gráfica refleja que 50\% de los varones también.

$\mathrm{Al}$ respeto, se puede indicar que ambos grupos tienen similitud en cuanto al mismo estilo de aprendizaje. Lo que demuestra que los estudiantes de ambos géneros perciben dos tipos de información:

La externa o sensitiva, que involucra el aspecto visual, auditivo y las sensaciones físicas. Esto demuestra que la visión y el oído juegan un papel importante en el aprendizaje del idioma, a través de videos e imágenes para el logro del aprendizaje, por ejemplo.

La interna o intuitiva, que puede ser lograda a través de lecturas comprensivas y la discusión de las ideas que refuercen el proceso de asimilación, acomodación, e integración de una nueva lengua en la estructura cognitiva de ambos géneros. 
En consecuencia, no hay diferencia en cuanto al estilo de aprendizaje sensitivo-intuitivo entre alumnos y alumnas.

Gráfica 3. Datos porcentuales del estilo de aprendizaje visual-verbal

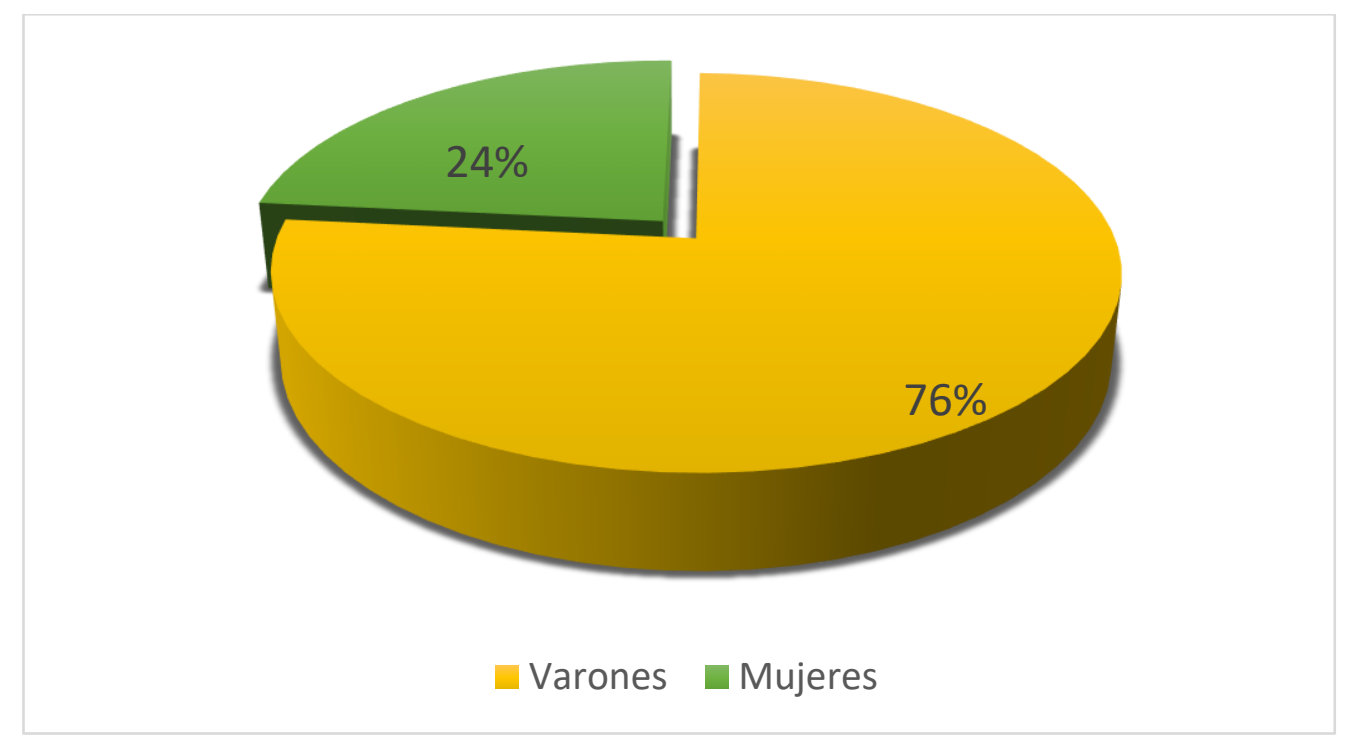

Fuente: Encuesta aplicada por el autor, basada en el cuestionario de Felder y Silverman.

En relación con la gráfica 3, se observa que el $24 \%$ de las alumnas tienen una tendencia no tan significativa hacia al estilo de aprendizaje visual-verbal, lo que demuestra, que este estilo de aprendizaje no presenta un grado de relevancia en la adquisición del idioma en el género femenino encuestado.

Respecto al grupo de hombres encuestados, se observa que un $76 \%$ de los alumnos manifiesta la importancia que tiene el estilo de aprendizaje visual-verbal en ellos. Esto indica que para el género masculino existe mejor internalización de los conocimientos lingüísticos del idioma a través de formatos visuales, imágenes, cuadros, gráficos, sonidos, expresión oral y escrita, símbolos y formulas, lo que les facilita a ellos una mejor comprensión en la adquisición de este aprendizaje.

Gráfica 4. Datos porcentuales del estilo de aprendizaje secuencial-global 


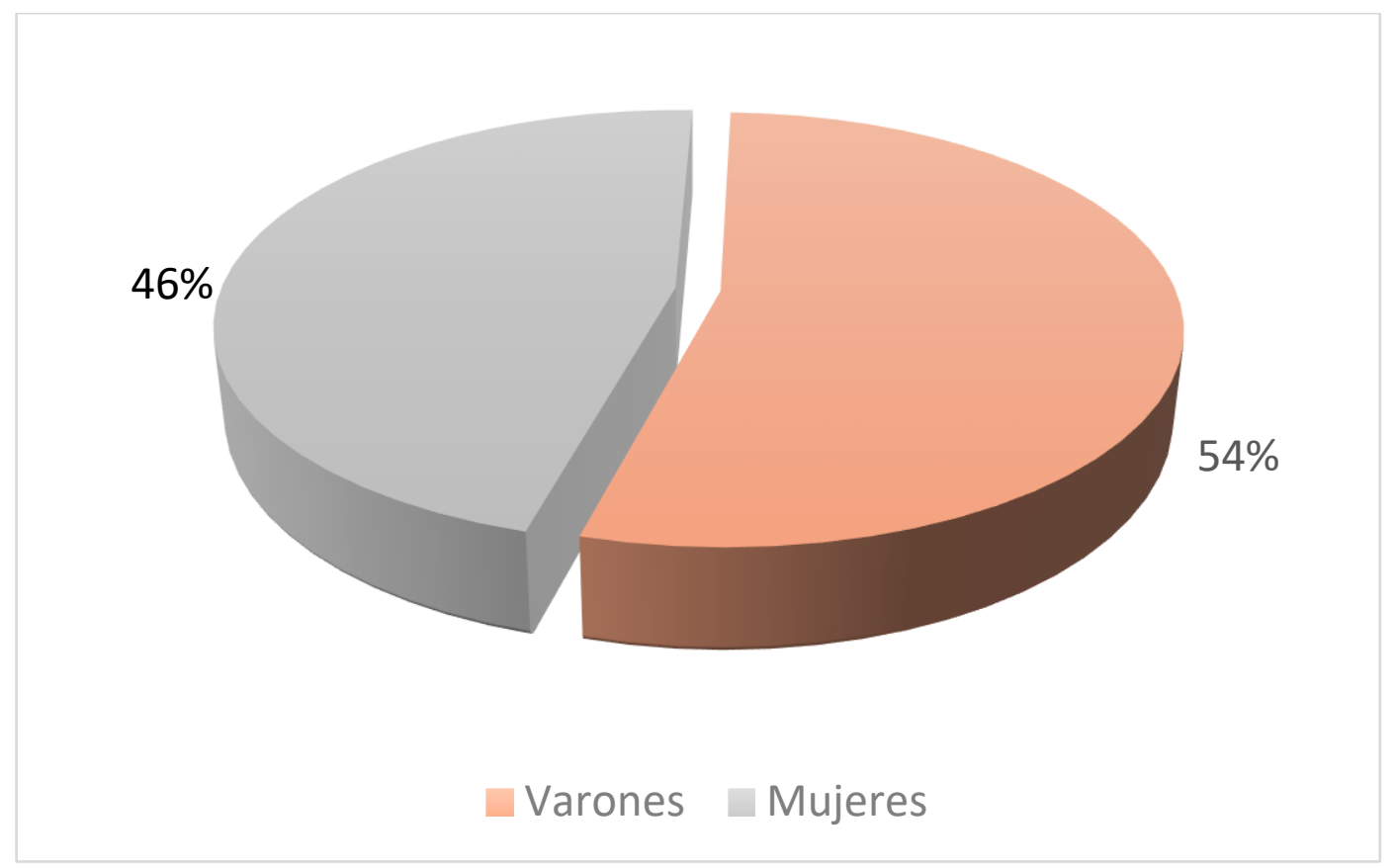

Fuente: Encueta aplicada por el autor, basada en el cuestionario de Felder y Silverman.

La gráfica 4 relacionada al estilo de aprendizaje secuencial-global en los estudiantes señala que, en el grupo de mujeres, solamente un $46 \%$ usa este estilo de aprendizaje en la adquisición del idioma inglés. Por otro lado, en el grupo de hombres, el 54\% manifiesta usar este estilo.

El resultado del estilo de aprendizaje secuencial-global indica que hay poca diferencia significa en cuanto al grupo de alumnas y alumnos en el aprendizaje de este idioma; ya que el estilo de aprendizaje secuencial-global implica un procedimiento de adquisición de la lengua inglesa de manera gradual y lógica, de tal manera que el idioma es aprendido de manera integral con niveles de complejidad que van desde de lo más simple a un mayor grado de dificultad.

\section{CONCLUSIONES}


Sin duda alguna, determinar los estilos de aprendizaje del idioma inglés en función de género en este trabajo sería una ambición inalcanzable y compleja, ya que la psicología ha aportado muchos estudios sobre cómo funciona la mente del ser humano con relación a los diferentes modos de adquirir el conocimiento desde diferentes perspectivas. Además, analizar el proceso de estilo de aprendizaje de un idioma a nivel de género requiere de muchos estudios en diferentes contextos, ya que la mente humana responde a las necesidades y exigencias del medio como un proceso de adaptación, y es una medición cualitativa difícil de cuantificar con exactitud por la complejidad de los individuos. Sin embargo, la intención ha sido presentar los resultados de un estudio representativo de estudiantes a nivel superior como una forma de contribuir al mejoramiento del proceso de enseñanza aprendizaje del idioma inglés.

Ofrecer los resultados de la investigación permite al docente que dicta la asignatura de inglés a nivel superior, tomar en cuenta los diferentes estilos de aprendizajes a la hora de planificar sus actividades y estrategias didácticas de enseñanza, y valorar la importancia de los estilos de aprendizaje de un segundo idioma. De esta manera, el docente podrá tomar en cuenta los procesos mentales del estudiante considerando los estilos de aprendizaje activo-reflexivo, sensorial-intuitivo, visual-verbal, y secuencial-global como forma de implementar cambios en la planificación curricular.

En el análisis de los resultados se puede constatar que existen diferencias en los estilos de aprendizaje del idioma inglés entre hombres y mujeres. El estilo activo-reflexivo en varones dio como resultado un $64 \%$, las mujeres obtuvieron un $36 \%$. En el estilo de aprendizaje visual-verbal, los varones presentaron mayor preferencia, con un $76 \%$, mientras que las mujeres, un 24\%; en lo que respecta al estilo de aprendizaje sensitivo-intuitivo, ambos grupos 
obtuvieron un $50 \%$. Los varones obtuvieron un $54 \%$ en aprendizaje secuencia-global, mientras que las mujeres obtuvieron un $46 \%$.

Este aporte está orientado a la reflexión del docente como mediador de conocimiento de una lengua extranjera que para algunos discentes es fácil de asimilar, pero para otros es más difícil de adquirir.

\section{REFERENCIA BIBLIOGRÁFICA}

Brown, H. Douglas (1994). Principles of Language Learning and Teaching. Ed. Printice, Hall Regents.

Chriler, Joan and Donald McCreary (2010). Handbook of Gender in Psychology: Volume 1: ED. Gender Research.

Elsner, Daniela y Viviane Johe (2016). Gender and Language Learning. Research and Practice. Ed.

Felder, R. y Silverman, L (1988). Estilos de Aprendizajes y enseñanza. Ingineering Education. Engineering Education, Vol 78, N78, 674-681.

Keefe, J.W. (1979). Learning style: An overview. NASSP's Student learning styles: Diagnosing and proscribing programs (pp. 1-17). Reston, VA. National

Salas, Raúl E. (2008). Estilos de aprendizaje a la luz de la Neurociencia. Aula abierta.

Wai, Chang and Suni Bhatt (2015). Culture and Foreign Education: Insights from Research and Implications for Practice. Boston.

Villanueva, M. L, I. Navarro (1997) Los Estilos de Aprendizaje de Lenguas. Ed. Universitat. Jaume

William, Lawrence (2015), Learning and Personaliy;ty: The Experienced of Introverted Reflective Learners in a world of Extroversts. Cambridge Scholar Publishing. 
